\title{
Fluorofenidone affects hepatic stellate cell activation in hepatic fibrosis by targeting the TGF-ß31/Smad and MAPK signaling pathways
}

\author{
YU PENG ${ }^{1}, \mathrm{LI} \mathrm{LI}^{2}$, XIN ZHANG ${ }^{3}$, MINGYAN XIE $^{2}, \mathrm{CONGYING} \mathrm{YANG}^{4}, \mathrm{SHATU}^{1}$, \\ HONG SHEN $^{5}$, GAOYUN HU ${ }^{6}$, LIJIAN TAO ${ }^{7}$ and HUIXIANG YANG ${ }^{1}$ \\ ${ }^{1}$ Department of Gastroenterology, Xiangya Hospital, Central South University, Changsha, Hunan 410008; \\ ${ }^{2}$ Department of Gastroenterology, The First People's Hospital of Changde City, Changde, Hunan 415000; \\ ${ }^{3}$ Department of General Practice, The First People's Hospital of Lianyungang, Lianyungang, Jiangsu 222000; \\ ${ }^{4}$ Department of Endoscopy Center, Hunan Cancer Hospital; ${ }^{5}$ Institute of Medical Sciences, Xiangya Hospital, \\ Central South University; ${ }^{6}$ Faculty of Pharmaceutical Sciences, Central South University, Changsha, Hunan 410000; \\ ${ }^{7}$ Department of Nephropathy, Xiangya Hospital, Central South University, Changsha, Hunan 410008, P.R. China
}

Received December 31, 2017; Accepted September 18, 2018

DOI: $10.3892 / \mathrm{etm} .2019 .7548$

\begin{abstract}
The aim of the present research was to study the therapeutic impacts of fluorofenidone (AKF-PD) on pig serum (PS)-induced liver fibrosis in rats and the complex molecular mechanisms of its effects on hepatic stellate cells (HSCs). Wistar rats were randomly divided into normal control, PS and PS/AKF-PD treatment groups. The activated human HSC LX-2 cell line was also treated with AKF-PD. The expression of collagen I and III, and $\alpha$-smooth muscle actin ( $\alpha$-SMA) was determined by immunohistochemical staining and reverse transcription-quantitative polymerase chain reaction (RT-qPCR). Western blotting and/or RT-qPCR analyses were used to determine the expression of transforming growth factor (TGF)- $\beta 1, \alpha$-SMA, collagen I, mothers against decapentaplegic homolog (Smad)-3, extracellular signal-regulated kinase (ERK)1/2, p38 mitogen-activated protein kinase (p38 MAPK) and c-Jun N-terminal kinase (JNK). AKF-PD attenuated the degree of hepatic fibrosis and liver injury in vivo, which was associated with the downregulation of collagen I and III, and $\alpha$-SMA at the mRNA and protein levels. In vitro, AKF-PD treatment significantly reduced the TGF- $\beta 1$-induced activation of HSCs, as determined by the reduction in collagen I and $\alpha$-SMA protein expression. The TGF- $\beta 1$-induced upregulation of the phosphorylation of Smad 3, ERK1/2, p38 and JNK was
\end{abstract}

Correspondence to: Professor Huixiang Yang, Department of Gastroenterology, Xiangya Hospital, Central South University, 87 Xiangya Road, Changsha, Hunan 410008, P.R. China

E-mail: yang_hx430@163.com

Key words: fluorofenidone, hepatic fibrosis, hepatic stellate cells, transforming growth factor- $\beta 1 /$ mothers against decapentaplegic homolog, extracellular signal-regulated kinase/mitogen-activated protein kinase attenuated by AKF-PD treatment. These findings suggested that AKF-PD attenuated the progression of hepatic fibrosis by suppressing HSCs activation via the TGF- $\beta 1 / \mathrm{Smad}$ and MAPK signaling pathways, and therefore that AKF-PD may be suitable for use as a novel therapeutic agent against liver fibrosis.

\section{Introduction}

Liver fibrosis is a reaction to chronic liver injury resulting from various etiological factors, including alcohol consumption, drug misuse, hepatitis B or C infection or toxic injury (1). Liver fibrosis is a globally widespread and substantial healthcare issue that results in end-stage liver diseases, including cirrhosis, and ultimately hepatocellular carcinoma. The process of liver fibrosis can be reversible, whereas cirrhosis, the result of end-stage fibrosis, is usually irreversible (2). Therefore, it is necessary to develop effective anti-fibrotic therapy for the treatment of fibrosis and hepatic injury.

The activation of hepatic stellate cells (HSCs) serves an important role in the initiation and development of liver fibrosis. During liver injury, quiescent HSCs are activated by stimulating factors or fibrogenic cytokines, including transforming growth factor (TGF)- $\beta 1$, platelet-derived growth factor (PDGF) and tumor necrosis factor (TNF)- $\alpha$ (3), which induce trans-differentiation into myofibroblast-like cells. This step is characterized by the appearance of procollagen-I and $\alpha$-smooth muscle actin (SMA), ultimately leading to the accumulation and increased output of collagen and extracellular matrix (ECM) components (4). Notably, TGF- $\beta 1$ is an extremely potent pro-fibrogenic cytokine associated with hepatic fibrosis in humans and other animals (5). TGF- $\beta 1$ acts though stimulating the mothers against decapentaplegic homolog (Smad)-dependent activation of Snail, and the Smad-independent induction of the mitogen-activated protein kinase (MAPK) pathway (6).

Research to identify safe anti-fibrotic agents is of high importance and is urgently required. A recently identified water-soluble pyridone agent with anti-fibrotic properties, 
fluorofenidone [1-(3-fluorophenyl)-5-methyl-2-(1H)-pyridone; AKF-PD], can attenuate liver $(7,8)$, renal (9), pulmonary (10) and cardiac fibrosis (11), and has been adopted in phase I clinical trials. However, its therapeutic benefits for hepatic fibrosis remain unclear. The aim of the present study was to research the anti-fibrotic effects of AKF-PD on liver fibrosis in rats and to elucidate the underlying mechanisms of the effect of AKF-PD on the activation of HSCs.

\section{Materials and methods}

Materials. LX-2 (12) HSCs [provided by Professor Scott L. Friedman (Icahn School of Medicine at Mount Sinai, New York, NY, USA)] were used in the present study. Dulbecco's modified Eagle's medium (DMEM) and fetal bovine serum (FBS) were purchased from Gibco (Thermo Fisher Scientific, Inc., Waltham, MA, USA). TRIzol for RNA extraction was purchased from Invitrogen; Thermo Fisher Scientific, Inc. A quantitative polymerase chain reaction (qPCR) kit (SYBR PremixEx Taq II) was purchased from Takara Bio, Inc. (Otsu, Japan). Antibodies against total extracellular signal-regulated kinase (ERK1/2; cat. no. 9102), p38 mitogen-activated protein kinase (p38 MAPK; cat. no. 9212), c-Jun N-terminal kinase (JNK; cat. no. 9252), and phosphorylated (p)-ERK (cat. no. 9101), p-p38 (cat. no. 9211) and p-JNK (cat. no. 4668) were purchased from Cell Signaling Technology, Inc. (Danvers, MA, USA). Antibodies against $\alpha$-SMA (cat. no. A5228) and $\beta$-actin (cat. no. A5441) were purchased from Sigma-Aldrich; Merck KGaA (Darmstadt, Germany). Antibodies against p-Smad-3 (cat. no. ab193297), collagen I (cat. no. ab34710), collagen III (cat. no. ab23746) and Smad-3 (cat. no. ab40854) were purchased from Abcam (Cambridge, UK). Recombinant human TGF- $\beta 1$ was purchased from PeproTech, Inc. (Rocky Hill, NJ, USA). Horseradish peroxide (HRP)-conjugated secondary antibodies for western blot analysis were purchased from Jackson ImmunoResearch Laboratories, Inc. (West Grove, PA, USA), and HRP-conjugated secondary antibodies for immunohistochemistry were obtained from Golden Bridge International, Inc. (Bothell, WA, USA). An enhanced chemiluminescence (ECL) kit for western blot analysis was purchased from GE Healthcare Life Sciences (Little Chalfont, UK). Pig serum (PS) was purchased from Beijing YuanHeng ShengMa Biology Technology Research Institute of Biotechnology (Beijing, China). AKF-PD was synthesized by Sunshine Lake Pharma Co., Ltd. (Dongguan, China; cat. no. 090601).

Animal model. Male albino Wistar rats weighing 120-150 g (5-6 weeks old) were obtained from Shanghai SLAC Laboratory Animal Co., Ltd. (Shanghai, China). Rats were bred and maintained in an air-conditioned environment (temperature $23-25^{\circ} \mathrm{C}$; humidity $50 \pm 2 \%$; $12 \mathrm{~h}$ light/dark cycle), with a commercial diet and water available ad libitum. The experimental protocol was approved by the Ethics Review Committee for Animal Experimentation of Central South University (Changsha, China), and all rats received humane care in compliance with the university's guidelines. For the purpose of the present study, rats were randomly divided into the following groups: Normal group $(n=15)$, PS model group $(n=15)$ and PS+AKF-PD treatment group $(n=15)$. Hepatic fibrosis was induced via intraperitoneal injections of $0.5 \mathrm{ml}$
PS twice weekly for 8 weeks (13), whereas the normal group received $0.5 \mathrm{ml}$ sterile saline. From the ninth week, the treatment group was administered AKF-PD intragastrically (240 mg/kg/day) once daily for 4 weeks. The normal and PS model groups were simultaneously administered with $0.5 \%$ carboxymethyl cellulose sodium intragastrically every day for 4 weeks. At the end of week 12, all rats were sacrificed. Livers were rapidly harvested, rinsed in cold saline and weighed while wet. A portion of liver was fixed (room temperature) in $10 \%$ neutral-buffered formalin for histopathology and the remaining tissue was stored at $-70^{\circ} \mathrm{C}$ until assayed.

Histological and immunohistochemical analysis. The formalin-fixed rat liver tissue was dehydrated in a graded alcohol series and embedded in paraffin. The paraffin sections (4- $\mu \mathrm{m}$ thick) were stained (room temperature for $10 \mathrm{~min}$ ) with hematoxylin and eosin or Masson's trichrome. To determine the degree of necroinflammatory liver injury, histological grading and the quantification of infiltrating inflammatory cells were blindly performed by an independent pathologist, as described previously $(14,15)$. To further analyze the degree of interstitial collagen deposition, Masson's trichrome-stained sections were graded as previously reported (16). 3,3'-Diaminobenzidine immunohistochemical staining was performed using the DAKO EnVision system (Dako; Agilent Technologies, Inc., Santa Clara, CA, USA), as previously detailed (17). Subsequent to blocking non-specific binding with the included blocking solution, the slides were incubated with primary antibodies against collagen I (1:100), collagen III (1:150) and $\alpha$-SMA (1:250) overnight at $4^{\circ} \mathrm{C}$, followed by the corresponding secondary antibodies (1:100) at room temperature for $1 \mathrm{~h}$. HRP-labeled polymer and a chromogen substrate were then employed to develop the staining. As a negative control, the primary antibody was replaced with PBS. Samples were visualized using light microscopy at a magnification of x 100. Given the homogeneity of the staining of the target proteins, the interstitial staining of collagen I, collagen III and $\alpha$-SMA was measured by a blinded observer using computerized morphometry (QWin 2.8 software; Leica Microsystems GmbH, Wetzlar, Germany) (18).

Analysis of $m R N A$ expression. Total RNA was isolated from the fresh liver tissue using TRIzol reagent, according to the manufacturer's protocol. First-strand cDNA was synthesized from $2 \mu \mathrm{g}$ total RNA in a $20-\mu 1$ reaction, using a ReverseAid first strand cDNA synthesis kit (Thermo Scientific Inc.) according to the manufacturer's protocol. Specific primers for collagen I, collagen III, $\alpha$-SMA, TGF- $\beta 1$ and $\beta$-actin were designed from their GenBank sequences (8) and synthesized by Bio Basic, Inc. (Markham, ON, Canada; Table I). qPCR quantitation for each target mRNA was performed on an ABI Model 7900 Detector (Applied Biosystems, Foster City, CA, USA) using the Takara qPCR kit. The thermocycling conditions were as follows: $95^{\circ} \mathrm{C}$ for $10 \mathrm{sec}$; followed by 40 cycles at $95^{\circ} \mathrm{C}$ for $5 \mathrm{sec}, 60^{\circ} \mathrm{C}$ for $30 \mathrm{sec}$; followed by $95^{\circ} \mathrm{C}$ for $15 \mathrm{sec}, 60^{\circ} \mathrm{C}$ for $15 \mathrm{sec}$ and $95^{\circ} \mathrm{C}$ for $15 \mathrm{sec}$ ). The amount of each mRNA in the samples was calculated from the standard curve and normalized to the $\beta$-actin mRNA. The comparative $2^{-\Delta \Delta \mathrm{Cq}}$ method was used for quantification and the results were expressed as fold-changes relative to the normal controls (19). 
Table I. Nucleotide sequences of the primers used for reverse transcription-quantitative polymerase chain reaction.

\begin{tabular}{lll}
\hline Gene & \multicolumn{1}{c}{ Forward (5'-3') } & \multicolumn{1}{c}{ Reverse $\left(5^{\prime}-3^{\prime}\right)$} \\
\hline Collagen I & TCAGGGGCGAAGGCAACAGT & TTGGGATGGAGGGAGTTTACACGA \\
Collagen III & CGTCCTGCAGGTAACAGTGGTTC & TGCTCCAGTTAGCCCTGCAA \\
$\alpha$-SMA & CTAAGGCCAACCGGGAGAAA & CCAGAGTCCAGCACAATACCA \\
TGF- $\beta 1$ & CAACAATTCCTGGCGTTACCTT & AAGCCCTGTATTCCGTCTCCTT \\
$\beta$-actin & GGAGATTACTGCCCTGGCTCCTA & GACTCATCGTACTCCTGCTTGCTG
\end{tabular}

SMA, smooth muscle actin; TGF, transforming growth factor.

Cell culture and treatment. LX-2 cells were maintained in DMEM supplemented with $10 \% \mathrm{FBS}, 100 \mathrm{U} / \mathrm{ml}$ penicillin and $100 \mu \mathrm{g} / \mathrm{ml}$ streptomycin (Invitrogen; Thermo Fisher Scientific, Inc.). The LX-2 cells were seeded onto 6-well culture plates to $60-70 \%$ confluence in DMEM containing 10\% FBS for $24-48 \mathrm{~h}$ at $37^{\circ} \mathrm{C}$. The medium was replaced with serum-free DMEM for $24 \mathrm{~h}$ prior to treatment with recombinant human TGF- $\beta 1$ at $37^{\circ} \mathrm{C}$ at a final concentration of $5 \mathrm{ng} / \mathrm{ml}$. For the detection of $\alpha$-SMA and collagen I, cells were pretreated at $37^{\circ} \mathrm{C}$ with $2 \mathrm{mM}$ AKF-PD as reported in a previous study (7), for $24 \mathrm{~h}$. TGF- $\beta 1$ was subsequently added to the medium, for $24 \mathrm{~h}$. Cells cultured in DMEM alone were used as a control. The cells were treated for $48 \mathrm{~h}$ at $37^{\circ} \mathrm{C}$, as determined by a preliminary experiment, prior to protein isolation. For detection of p-Smad-3, p-ERK1/2, p38 MAPK and p-JNK, cells were pretreated with $2 \mathrm{mM}$ AKF-PD for $24 \mathrm{~h}$ at $37^{\circ} \mathrm{C}$, and TGF- $\beta 1(5 \mathrm{ng} / \mathrm{ml})$ was subsequently added to the medium at $5,15,30$ and 60 min prior to the collection of cellular proteins. Each experiment was replicated three times.

Western blot analysis. Total protein $(30 \mu \mathrm{g})$ from the fresh liver tissue or cultured cells was extracted using sodium dodecyl sulfate (Sigma-Aldrich; Merck KGaA; cat. no. L3771) and determined using a Pierce ${ }^{\mathrm{TM}}$ BCA Protein assay kit (cat. no. 23227; Thermo Fisher Scientific Inc.). Samples (20 $\mu \mathrm{g}$ per lane) were separated by 8 or $10 \%$ SDS-PAGE under reducing conditions, and transferred onto polyvinylidene difluoride membranes (EMD Millipore, Billerica, MA, USA). Non-specific binding was blocked with TBS-T buffer [10 mM Tris/HCl, $150 \mathrm{mM}$ $\mathrm{NaCl}, 0.1 \%$ (v/v) Tween 20, pH 7.6] containing 5\% (w/v) skimmed milk for $1 \mathrm{~h}$ at room temperature. The membranes were incubated overnight at $4^{\circ} \mathrm{C}$ with primary antibodies against $\alpha$-SMA (dilution, 1:2,500), collagen I (dilution, 1:1,000), p-ERK (dilution, 1:1,000), p-p38 (dilution, 1:1,000), p-JNK (dilution, 1:1,000), p-Smad-3 (dilution, 1:1,000), ERK (dilution, 1:1,000), p38 (dilution, 1:1,000), JNK (dilution, 1:1,000), Smad-3 (dilution, $1: 1,000)$ and $\beta$-actin (dilution, 1:5,000), and were subsequently incubated with HRP-conjugated secondary antibodies (dilution, 1:5,000) for $1 \mathrm{~h}$ at room temperature. The bands were visualized using an ECL kit and quantified using Bandscan 5.0 software (Glyko, Inc., Novato, CA, USA). Phosphorylated proteins were normalized to the expression of the total protein and the loading control.

Statistical analysis. Data are expressed as the mean + standard deviation. Statistical analyses were performed using SPSS 19.0 software (IBM Corp., Armonk, NY, USA). The comparison among groups was made with a one-way analysis of variance (ANOVA). Multiple-comparison tests were applied only when a significant difference was determined by ANOVA followed by an LSD post-hoc test. $\mathrm{P}<0.05$ was considered to indicate a statistically significant difference.

\section{Results}

AKF-PD ameliorates the histological injury induced by PS. As demonstrated in Fig. 1A, PS-gavaged rats displayed impaired hepatic lobules, a reduced quantity of hepatic sinusoids, wide hemorrhagic necrosis and lobular architecture with narrow strips of reticulin connecting central zones. AKF-PD administration at the concentration of $240 \mathrm{mg} / \mathrm{kg} / \mathrm{day}$ attenuated these pathological modifications $(\mathrm{P}<0.05)$. Although the accumulation of collagen fibers between the portal region and pseudolobules was augmented in the PS model group, AKF-PD therapy significantly attenuated the increase in collagen expression $(\mathrm{P}<0.05$; Fig. 1B).

AKF-PD attenuates ECM deposition and HSCs activation in rat fibrotic liver. With respect to the deposition of collagen types I and III, the main ECM components of the fibrotic liver, and $\alpha$-SMA, a marker for HSC activation, no positive staining was identified in the control rat liver tissue (Fig. 2). The PS-injured liver exhibited a notable expression of collagen type I, collagen type III and $\alpha$-SMA, which was significantly suppressed by AKF-PD therapy ( $\mathrm{P}<0.05$; Fig. 2). Furthermore, these observations were confirmed by the RT-qPCR analysis of collagen type I, collagen type III and $\alpha$-SMA mRNA expression in liver tissue $(\mathrm{P}<0.05$; Fig. 3A-C).

The expression of TGF- $\beta 1$ is decreased by AKF-PD in fibrotic liver tissue. TGF- $\beta 1$ is a central mediator of liver fibrosis. The upregulated expression of TGF- $\beta 1$ has been detected in experimental liver fibrosis models (20). Therefore, it was considered whether AKF-PD diminished the expression of TGF- $\beta 1$. It was revealed that the expression of TGF- $\beta 1$ was notably increased following PS treatment, and that AKF-PD significantly attenuated the expression of TGF- $\beta 1$ ( $\mathrm{P}<0.05$; Fig. 3D).

AKF-PD alleviates TGF- $\beta 1$-induced $\alpha$-SMA and collagen I expression in HSCs. Having demonstrated that AKF-PD can effectively attenuate liver fibrosis in experimental animals, the cellular mechanism by which AKF-PD influences HSCs, the central cell type associated with liver fibrosis, was considered in 
A
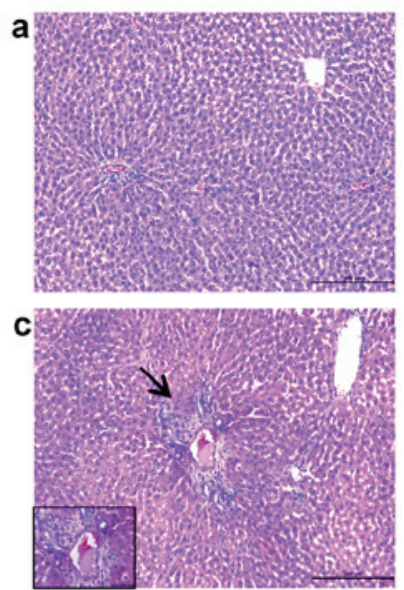
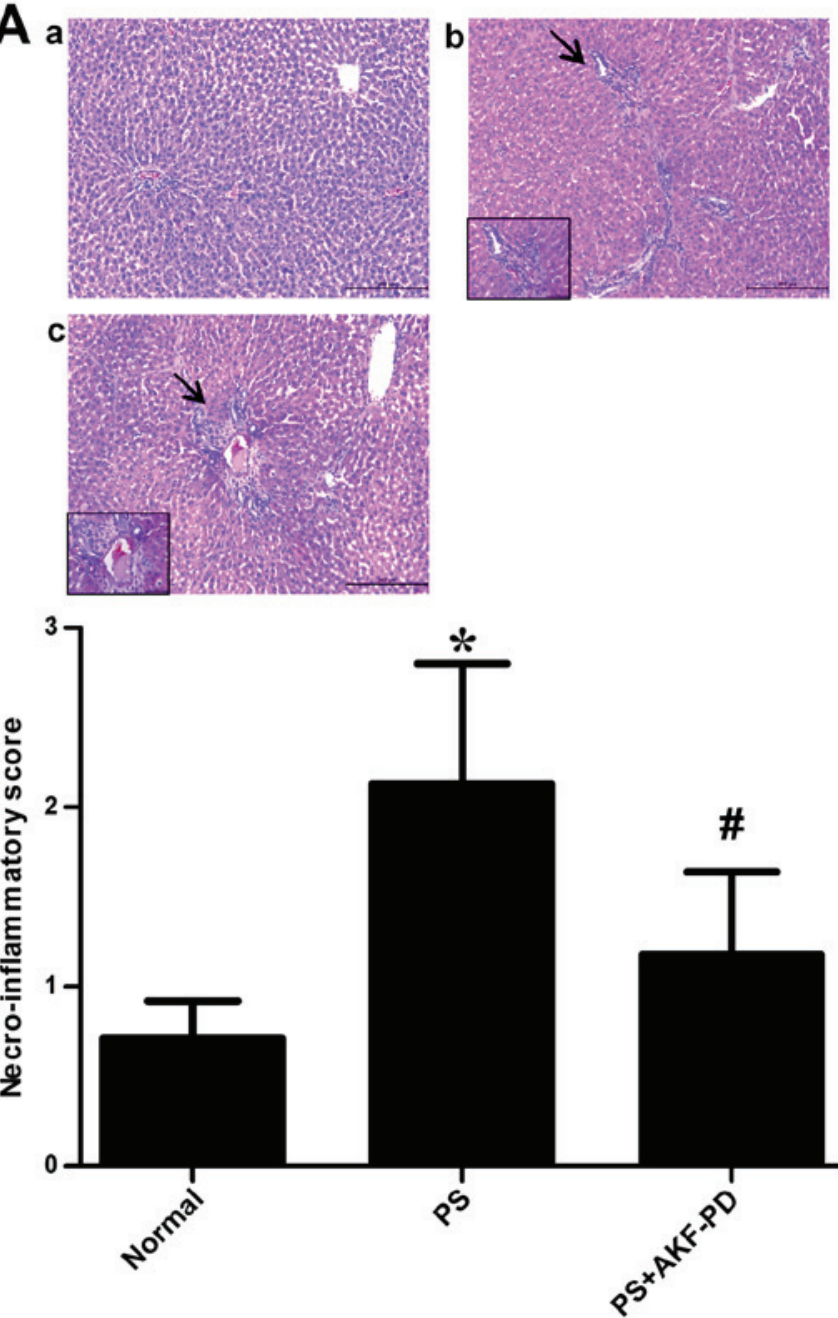

B
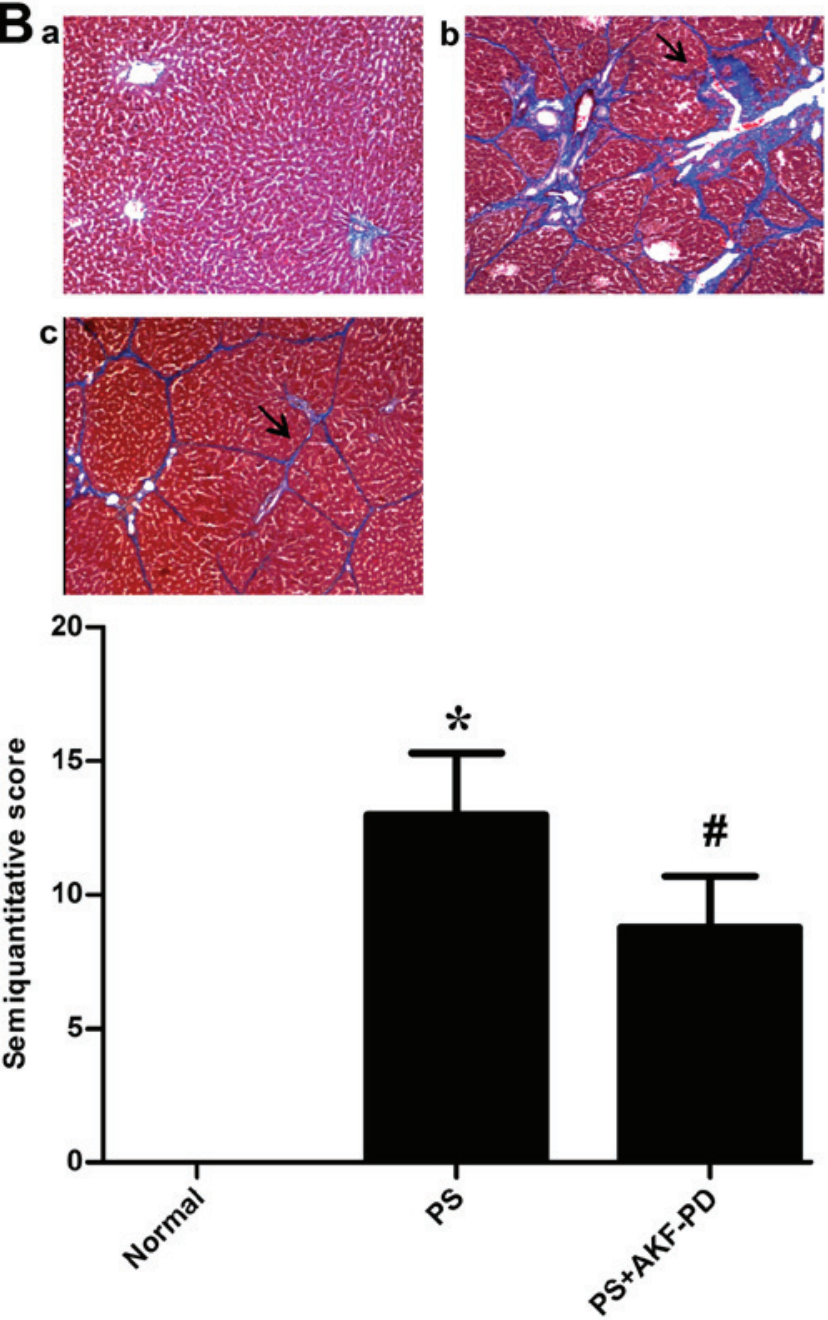

Figure 1. AKF-PD improves the histological injuries induced by pig serum. (A) Representative histological images of hematoxylin and eosin-stained rat liver tissue in the (a) normal, (b) PS and (c) PS+AKF-PD groups, respectively. Arrows indicate impaired hepatic lobules, a reduced quantity of hepatic sinusoids, wide hemorrhagic necrosis and lobular architecture with narrow strips of reticulin connecting central zones. Magnification, $\mathrm{x} 200$. Columns indicate the necroinflammatory score of liver fibrosis for each group. (B) Representative images of Masson-stained collagen deposition in rat livers in the (a) normal, (b) PS and (c) PS+AKF-PD groups, respectively. Arrows indicate the accumulation of collagen fibers between the portal region and pseudolobules. Magnification, x100. Columns indicate the semi-quantitative score for liver fibrosis in each group. Data are expressed as the mean \pm standard deviation $\left(15\right.$ rats/group). ${ }^{*}<0.05$ vs. normal group; ${ }^{\text {P }}<0.05$ vs. PS group. AKF-PD, fluorofenidone; PS, pig serum.

the LX-2 cell line. Following the incubation of LX-2 cells with AKF-PD, the expression of $\alpha$-SMA initiated by TGF- $\beta 1$ was significantly decreased $(\mathrm{P}<0.05$; Fig. $4 \mathrm{~A})$. The protein expression of the fibrosis marker collagen I was also detected. As indicated in Fig. 4B, a 2.6-fold increase in collagen I expression was induced by TGF- $\beta 1$, which was suppressed by AKF-PD to a variable extent $(\mathrm{P}<0.05)$.

AKF-PD inhibits the TGF- $\beta 1 /$ Smad and MAPK pathways in $H S C s$. To further identify the molecular mechanisms responsible for the inhibitory effects of AKF-PD on TGF- $\beta 1$-induced HSC activation, the effects of AKF-PD on TGF- $\beta 1$-induced TGF- $\beta 1 /$ Smad and MAPK pathways in LX-2 cells were analyzed. The time course of the activation of Smad-3, ERK, p38 and JNK in reaction to TGF- $\beta 1$ was detected in LX- 2 cells. Significant increases in p-Smad-3, p-ERK, p-p38 and p-JNK were observed as early as $15 \mathrm{~min}$ following TGF- $\beta 1$ treatment (Fig. 5). The levels of p-Smad3, p-ERK, p-p38 and p-JNK were markedly decreased by co-treatment with AKF-PD. Therefore, these data suggest that AKF-PD inhibits TGF- $\beta 1$-induced HSCs activation by targeting the TGF- $\beta 1 / \mathrm{Smad}$ and MAPK signaling pathways.

\section{Discussion}

The inordinate accumulation of ECM proteins such as collagen leads to liver fibrosis, which is associated with the majority of chronic liver diseases. Advanced stages of liver dysfunction and cirrhosis result in liver fibrosis (21). However, despite the advancements in knowledge regarding the molecular and cellular mechanisms of liver fibrosis and cirrhosis, the available anti-fibrotic treatment options are insufficient. In certain cases, causes including alcohol-induced liver fibrosis may further the progression of liver fibrosis, even following the withdrawl of the causative agent (alcohol) (22).

In the present study, the anti-fibrotic effect of AKF-PD and its potential mechanisms were identified: AKF-PD was revealed to reduce the number of activated HSCs, as demonstrated by the 
$\mathbf{A}_{\mathrm{a}}$
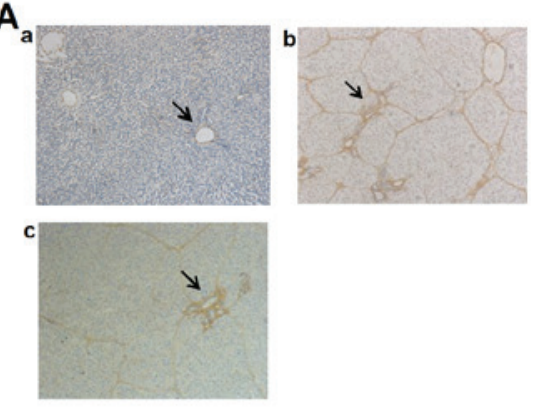

$\mathbf{B}_{\mathrm{a}}$
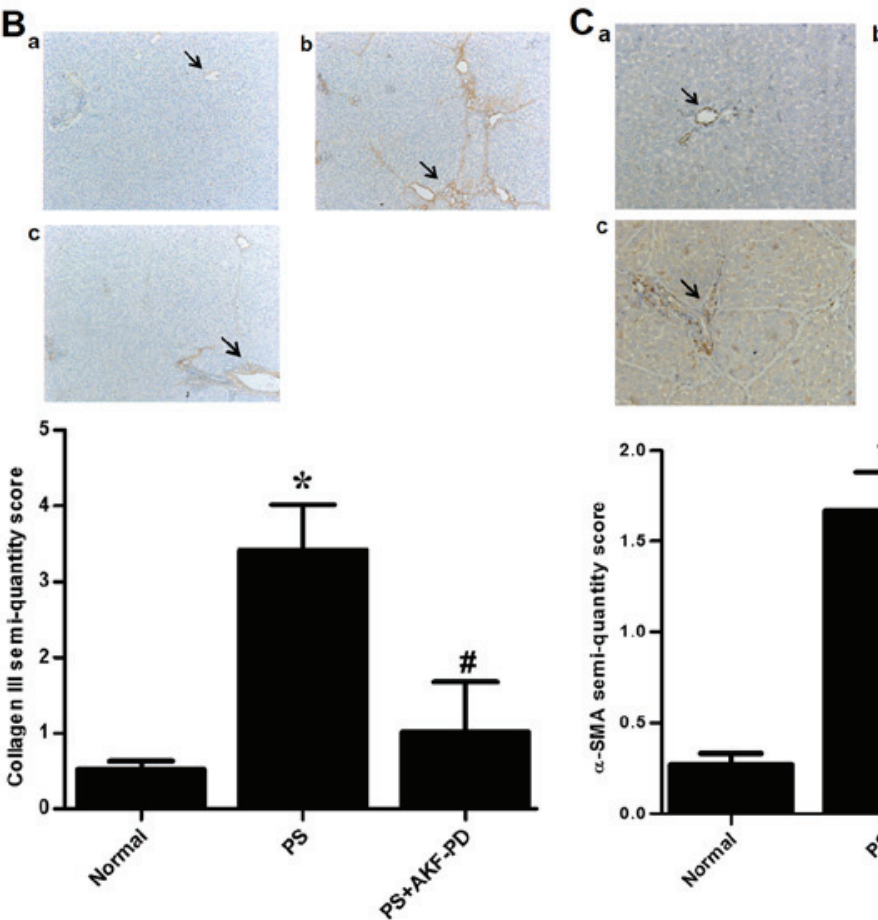
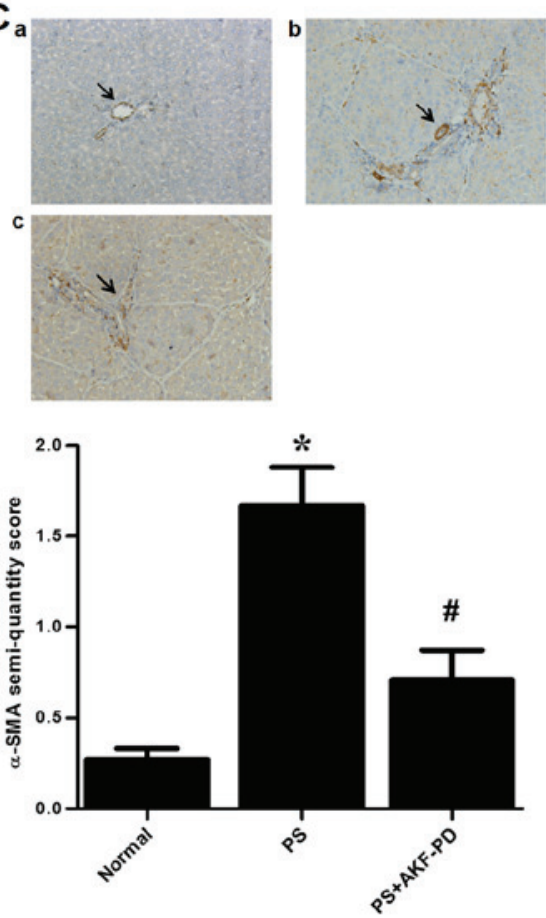

Figure 2. AKF-PD attenuates ECM deposition and HSC activation in rat fibrotic liver tissue. Immunohistochemical staining for (A) collagen I, (B) collagen III and (C) $\alpha$-SMA in liver tissue in the (a) normal, (b) PS and (c) PS+AKF-PD groups are presented (magnification, x100). Arrows indicate the deposition of collagen types I and III, the main ECM components of the fibrotic liver, and $\alpha$-SMA, a marker for HSC activation. Columns indicate the semi-quantified scores for collagen I, collagen III and $\alpha$-SMA expression. Data were expressed as the mean \pm standard deviation $\left(6\right.$ rats $/$ group). ${ }^{*} \mathrm{P}<0.05$ vs. normal group; ${ }^{\prime} \mathrm{P}<0.05$ vs. PS group. AKF-PD, fluorofenidone; ECM, extracellular matrix; SMA, smooth muscle actin; PS, pig serum; HSC, hepatic stellate cell.
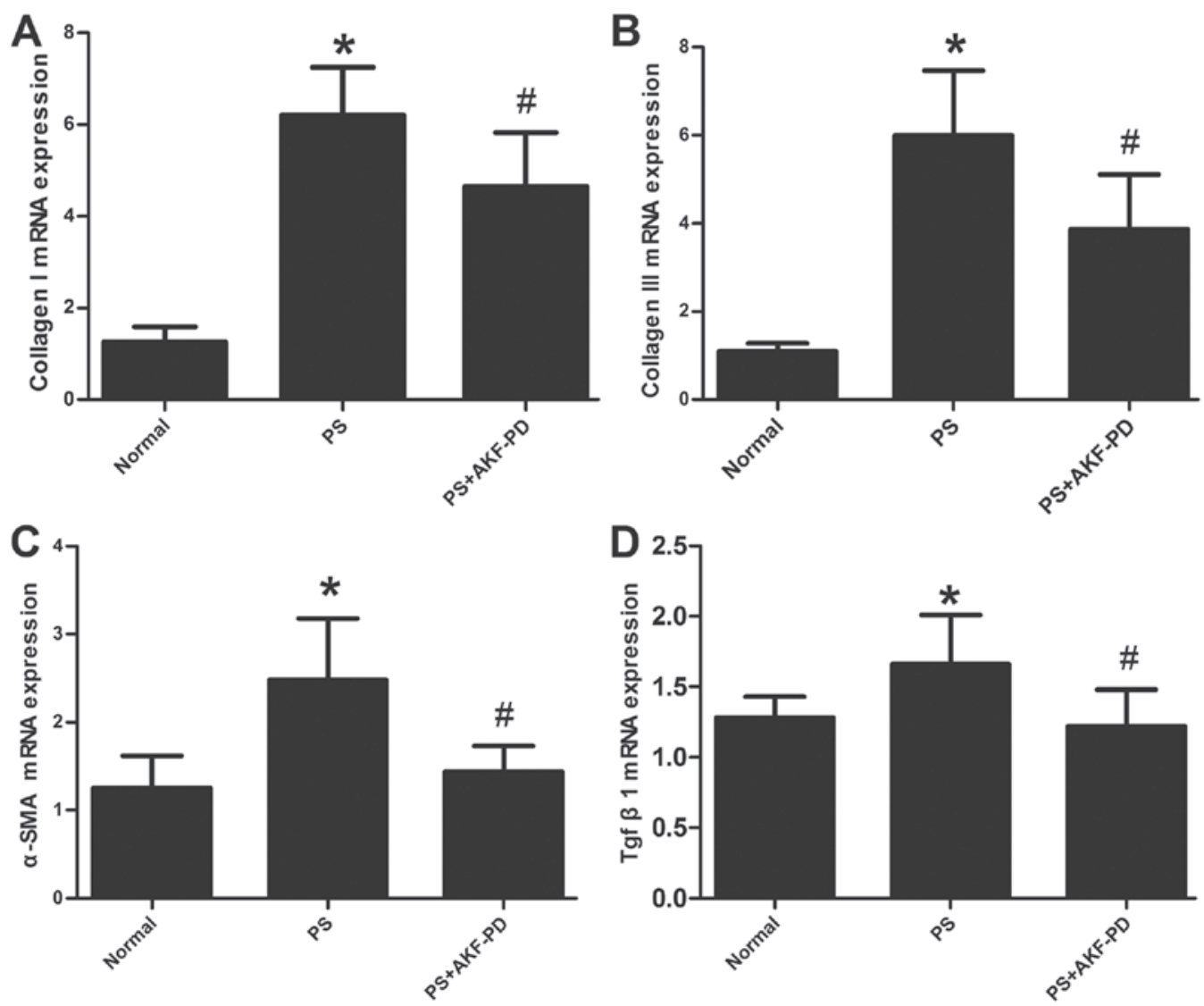

Figure 3. AKF-PD decreases the expression of collagen I, III, $\alpha$-SMA and TGF- $\beta 1$ in fibrotic liver tissue. Reverse transcription-quantitative polymerase chain reaction was used to determine the levels of (A) collagen I, (B) collagen III, (C) $\alpha$-SMA and (D) TGF- $\beta 1$ mRNA expression following AKF-PD treatment. Data are expressed as the mean \pm standard deviation (6 rats/group). ${ }^{~} \mathrm{P}<0.05$ vs. normal group; ${ }^{\#} \mathrm{P}<0.05$ vs. PS group. AKF-PD, fluorofenidone; SMA, smooth muscle actin; TGF, transforming growth factor; PS, pig serum. 

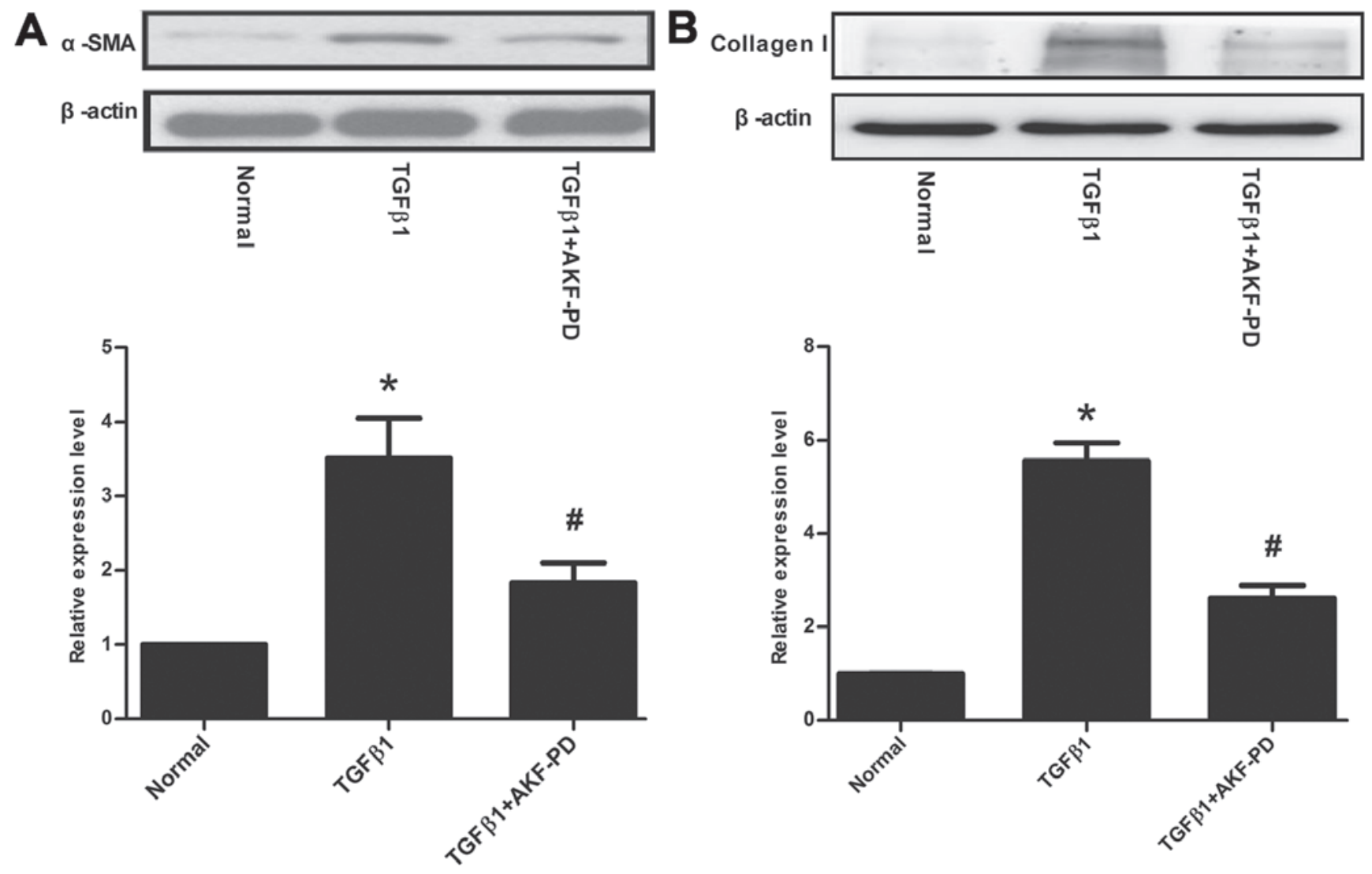

Figure 4. AKF-PD alleviates TGF- $\beta 1$-induced $\alpha$-SMA and collagen I expression in HSCs. AKF-PD decreases TGF- $\beta 1$-induced (A) $\alpha$-SMA and (B) collagen I expression in HSCs, as determined by western blot analysis. Untreated cells were used as a negative control. " $\mathrm{P}<0.05$ vs. control group; ${ }^{*} \mathrm{P}<0.05$ vs. TGF- 31 -treated group. AKF-PD, fluorofenidone; TGF, transforming growth factor; SMA, smooth muscle actin; HSC, hepatic stellate cell; PS, pig serum.

decrease in $\alpha$-SMA (a myofibroblast marker) and ECM protein expression, which was mediated at least partly by attenuating the TGF- $\beta 1$-induced upregulation of the TGF- $\beta /$ Smad and MAPK pathways.

The progression of liver fibrosis is a dynamic process comprising several types of cells in the hepatic sinusoids. It is characterized by disturbed hepatic architecture and the accumulation of ECM proteins. The activation of HSCs in response to hepatic injury is a key process in hepatic fibrogenesis, comprising the transformation of quiescent vitamin A-rich cells into proliferative, fibrogenic and contractile myofibroblasts (23). $\alpha$-SMA is a marker for the detection of activated HSCs during fibrogenesis (24). The $\mathrm{CCl}_{4}$ - and dimethylnitrosamine-induced liver fibrosis models are chemically induced injuries, whereas PS immunologically induced injury is more likely to simulate hepatic fibrosis, caused by hepatitis B (13). In the present study, the oral administration of AKF-PD to rats hindered PS-induced $\alpha$-SMA expression and collagen type I and III deposition, as evaluated by immunohistochemistry, a result that was also identified at the mRNA level. These data implied that PS-induced liver fibrosis can be attenuated by AKF-PD.

Hepatic fibrosis is characterized by the inordinate yield and deposition of ECM proteins, resulting in the devastation of the ordinary hepatic parenchyma and interruption of the liver structure $(25,26)$. Collagen types I and III and other ECM proteins can be excessively deposited, thereby causing organ malfunction and failure (27). The regulation of collagen I and III expression has been extensively studied to determine the mechanism of fibrosis (28). TGF- $\beta 1$ is a central mediator of HSC activation and ECM protein accumulation, as it gives rise to fibrosis. The secretion and activation of TGF- $\beta 1$ stimulates the synthesis of various ECM components, including collagen I and III (29). TGF- $\beta 1$ expression is markedly augmented in cirrhotic liver tissue; it is a potent inducer of stellate collagen production and cell proliferation (30). In the present study, TGF- $\beta 1$ expression was reduced by AKF-PD in PS-induced liver fibrosis, suggesting that the anti-fibrotic effects of AKF-PD may be mediated through the suppression of TGF- $\beta 1$.

HSCs are activated by a variety of cytokines, including PDGF and TGF- $\beta 1$, which are produced by endothelial cells, hepatocytes and Kupffer cells (29). PDGF is the most potent proliferative cytokine in HSCs, whereas TGF- $\beta 1$ is the strongest pro-fibrotic factor $(31,32)$. The transformation of HSCs into myofibroblasts can be promoted by TGF- $\beta 1$; the synthesis and degradation of the ECM can also be stimulated and inhibited by TGF- $\beta 1$ (33). In the present study, AKF-PD attenuated the TGF- $\beta 1$-induced expression of collagen I and $\alpha$-SMA in vitro, suggesting that AKF-PD decreases the extent of liver fibrosis by suppressing TGF- $\beta 1$.

TGF- $\beta 1$ signaling is transduced through the sequential activation of its two serine/threonine kinase receptors (TGF- $\beta$ type I receptor and TGF- $\beta$ type II receptor), which in turn phosphorylate Smad-3 or Smad-2 to induce nuclear translocation (34). Smad-3 regulates the transcription of fibrogenic genes, including $\alpha$-SMA and pro-collagen type I, which are important for the production of ECM proteins to repair damaged 


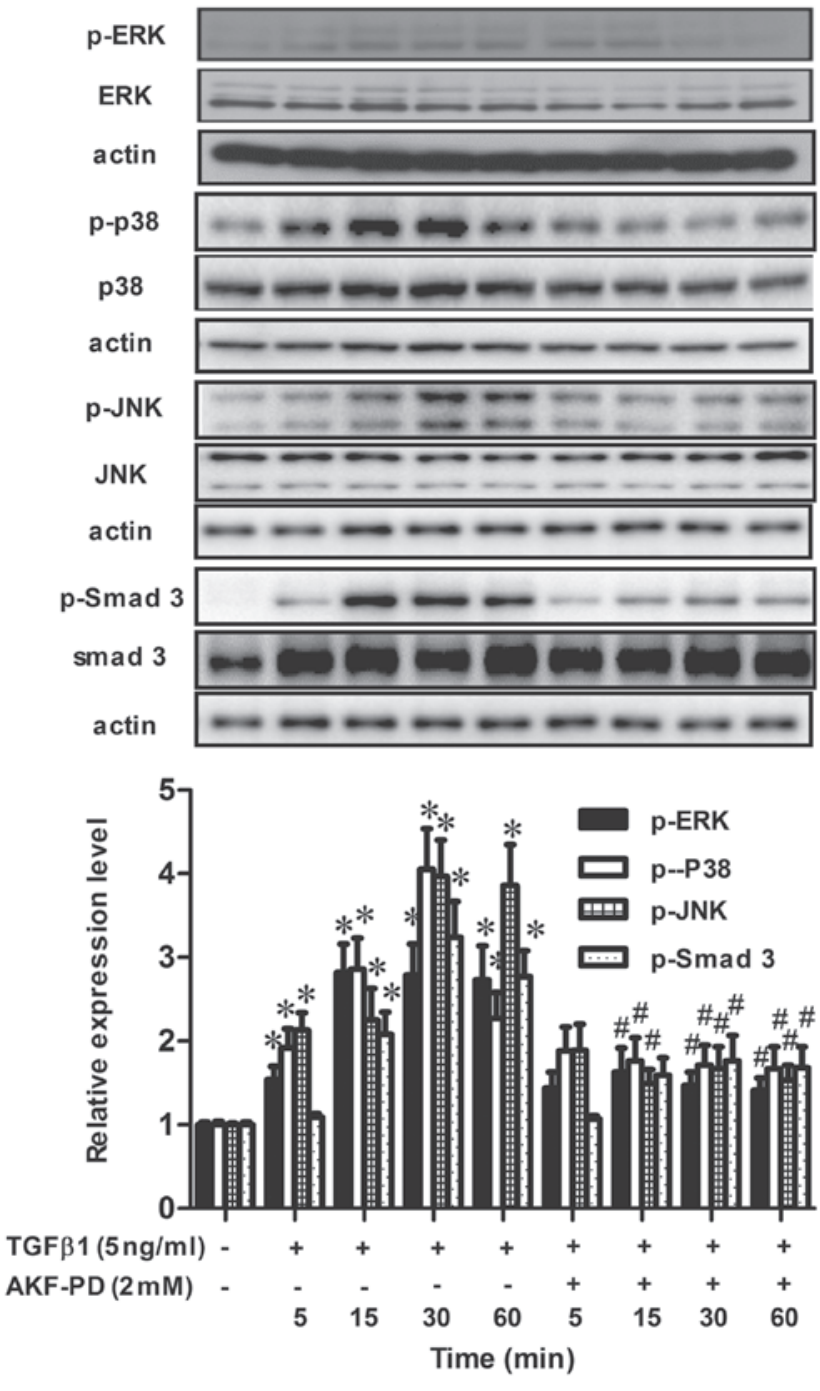

Figure 5. AKF-PD inhibits the TGF- $\beta 1 /$ Smad and mitogen-activated protein kinase pathways in HSCs. AKF-PD decreases the phosphorylation of Smad-3, ERK, p38 and JNK in TGF- $\beta 1$-induced HSCs as determined by western blot analysis. Untreated cells were used as a negative control. " $\mathrm{P}<0.05$ vs control group; ${ }^{\#} \mathrm{P}<0.05$ vs. TGF- $\beta 1$-treated group. AKF-PD, fluorofenidone TGF, transforming growth factor; Smad, mothers against decapentaplegic homolog; HSC, hepatic stellate cell; ERK, extracellular signal-regulated kinase; JNK, c-Jun N-terminal kinase; p, phosphorylated.

tissues (35). Therefore, the inhibition of TGF- $\beta 1$ signaling in HSCs is a therapeutic target for inhibiting the initiation and progression of hepatic fibrosis. TGF- $\beta 1$ is the primary isoform associated with liver fibrosis. The results of the present study revealed that treatment with $5 \mathrm{ng} / \mathrm{ml}$ TGF- $\beta 1$ activated HSCs, as confirmed by the increase in $\alpha$-SMA and collagen I protein expression. Furthermore, the signaling pathway activated by TGF- $\beta 1$ affects the type I receptor-mediated phosphorylation of Smad-3 (36), which was also suppressed by AKF-PD. These data suggest that AKF-PD blocks fibrogenesis via an effect on the TGF- $\beta /$ Smad signaling pathway.

Smad-3 was demonstrated to be an effector of TGF- $\beta 1$-induced fibrosis (37). Additionally, a number of non-Smad signaling molecules, including ERK, p38, JNK, and phosphatidylinositol-3-kinase have previously been identified as mediators of TGF- $\beta 1$-induced fibrosis (38). Smads and the canonical Smad transcription factor pathway have been the focus of previous research (39). The linker domains of $\mathrm{Smad} 2 / 3$ can act as sensors of Smad independent or non-Smad signal transduction cascades. There are several possible ERK phosphorylation sites within the linker region domains of Smad2 and Smad3 (40). In addition to the Smad-mediated canonical TGF- $\beta 1$ signaling pathway, the ERK, p38 MAPK and JNK signaling pathways can also be activated by TGF- $\beta 1$ (41). Furthermore, TGF- $\beta 1$ can augment collagen type I expression through the MAPK and Akt pathways (42), and the signaling pathway activated by TGF- $\beta 1$ comprises non-Smad signaling molecules, including ERK, p38 and JNK, which were also suppressed by AKF-PD. These data indicated that AKF-PD blocks fibrogenesis through the MAPK signaling pathway.

Considering the ability of AKF-PD to ameliorate liver fibrosis, the present study investigated its effect on TGF- $\beta 1$-mediated HSC activation in LX-2 cells and on the fibrogenesis induced by PS in rats. The present study demonstrated that AKF-PD inhibits PS-stimulated liver fibrosis and suppresses TGF- $\beta 1$-mediated HSCs activation. The impacts of AKF-PD on HSCs activation through the TGF- $\beta 1 /$ Smad and MAPK pathways, which serve crucial roles in fibrogenesis, were also identified. The results of the present study may inform a novel therapeutic strategy for preventing liver fibrosis and other liver diseases.

\section{Acknowledgements}

The authors would like to thank Professor Scott L. Friedman (Icahn School of Medicine at Mount Sinai, New York, NY, USA) for donating a sample of the LX-2 cell line.

\section{Funding}

The present study was supported by the grants from National Natural Science Foundation of China (grant nos. 81400642, 81370547 and 81673499).

\section{Availability of data and materials}

All data generated or analyzed during this study are included in this published article.

\section{Authors' contributions}

YP, LT and HY conceived and designed the current study. YP, LL, XZ, MX, CY and ST performed the experiments. YP analyzed the data. YP, HS and GH interpreted the results of experimentation. YP prepared the figures and drafted the manuscript. YP and HS edited and revised manuscript. YP, HY and LT approved the final version of manuscript. All authors read and approved the manuscript.

\section{Ethics approval and consent to participate}

The present protocol was approved by the Ethics Review Committee for Animal Experimentation of Central South University (Changsha, China).

\section{Patient consent for publication}

Not applicable. 


\section{Competing interests}

The authors declare that they have no competing interests.

\section{References}

1. Bataller R and Brenner DA: Liver fibrosis. J Clin Invest 115 209-218, 2005.

2. Friedman SL and Bansal MB: Reversal of hepatic fibrosis e fact or fantasy? Hepatology 43(2 Suppl 1): S82-S88, 2006.

3. Parola M and Robino G: Oxidative stress-related molecules and liver fibrosis. J Hepatol 35: 297-306, 2001.

4. Balta C, Herman H, Boldura OM, Gasca I, Rosu M, Ardelean A and Hermenean A: Chrysin attenuates liver fibrosis and hepatic stellate cell activation through TGF-b/Smad signaling pathway. Chem Biol Interact 240: 94-101, 2015.

5. Williams E and Iredale J: Hepatic regeneration and TGF-beta: Growing to a prosperous perfection. Gut 46: 593-594, 2000.

6. Xu J, Lamouille S and Derynck R: TGF-beta-induced epithelial to mesenchymal transition. Cell Res 19: 156-172, 2009.

7. Peng Y, Yang H, Wang N, Ouyang Y, Yi Y, Liao L, Shen H, Hu G, Wang $Z$ and Tao L: Fluorofenidone attenuates hepatic fibrosis by suppressing the proliferation and activation of hepatic stellate cells. Am J Physiol Gastrointest Liver Physiol 306: 253-263, 2014.

8. Peng Y, Yang H, Zhu T, Zhao M, Deng Y, Liu B, Shen H, Hu G Wang $Z$ and Tao L: The antihepatic fibrotic effects of fluorofenidone via MAPK signalling pathways. Eur J Clin Invest 43: 358-368, 2013

9. Tang Y, Zhang F, Huang L, Yuan Q, Qin J, Li B, Wang N, Xie Y, Wang L, Wang W, et al: The protective mechanism of fluorofenidone in renal interstitial inflammation and fibrosis. Am J Med Sci 350: 195-203, 2015.

10. Song C, He L, Zhang J, Ma H, Yuan X, Hu G, Tao L, Zhang J and Meng J: Fluorofenidone attenuates pulmonary inflammation and fibrosis via inhibiting the activation of NALP3 inflammasome

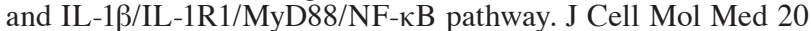
2064-2077, 2016

11. Chen LX, Yang K, Sun M, Chen Q, Wang ZH, Hu GY and Tao LJ: Fluorofenidone inhibits transforming growth factor-betal-induced cardiac myofibroblast differentiation. Pharmazie 67: 452-456, 2012.

12. Xu L, Hui AY, Albanis E, Arthur MJ, O'Byrne SM, Blaner WS, Mukherjee P, Friedman SL and Eng FJ: Human hepatic stellate cell lines, LX-1 and LX-2: New tools for analysis of hepatic fibrosis. Gut 54: 142-151, 2005.

13. Paronetto F and Popper H: Chronic liver injury induced by immunologic reactions Cirrhosis following immunization with heterologous sera. Am J Pathol 49: 1087-1101, 1966.

14. Benedetti A, Di Sario A, Casini A, Ridolfi F, Bendia E, Pigini P, Tonnini C, D'Ambrosio L, Feliciangeli G, Macarri G and Svegliati-Baroni G: Inhibition of the $\mathrm{NA}(+) / \mathrm{H}(+)$ exchanger reduces rat hepatic stellate cell activity and liver fibrosis: An in vitro and in vivo study. Gastroenterology 120: 545-556, 2001.

15. Di Sario A, Bendia E, Taffetani S, Marzioni M, Candelaresi C, Pigini P, Schindler U, Kleemann HW, Trozzi L, Macarri G and Benedetti A: Selective Na+/H+ exchange inhibition by cariporide reduces liver fibrosis in the rat. Hepatology 37: 256-266, 2003.

16. Chevallier M, Guerret S, Chossegros P, Gerard F and Grimaud JA: A histological semi-quantitative scoring system for evaluation of hepatic fibrosis in needle liver biopsy specimens: Comparison with morphometric studies. Hepatology 20: 349-355, 1994.

17. Pei H, Zhu H, Zeng S, Li Y, Yang H, Shen L, Chen J, Zeng L, Fan J, Li X, et al: Proteome analysis and tissue microarray for profiling protein markers associated with lymph node metastasis in colorectal cancer. J Proteome Res 6: 2495-2501, 2007.

18. Broekema M, Harmsen MC, van Luyn MJ, Koerts JA, Petersen AH, van Kooten TG, van Goor H, Navis G and Popa ER Bone marrow-derived myofibroblasts contribute to the renal interstitial myofibroblast population and produce procollagen I after ischemia/reperfusion in rats. J Am Soc Nephrol 18: 165-175, 2007.

19. WinerJ,Jung CK, Shackel Iand WilliamsPM: Developmentand validation of real-time quantitative reverse transcriptase-polymerase chain reaction for monitoring gene expression in cardiacmyocytes in vitro. Anal Biochem 270: 41-49, 1999.

20. Tsui HT, Wea LL, Zi HC, Lee YJ, Shie MS, Lee KF, Shen CH and Kuo HC: Moniliformediquinone as a potential therapeutic agent, inactivation of hepatic stellate cell and inhibition of liver fibrosis in vivo. J Transl Med 14: 263, 2016.

21. Ferrell LD and Kakar S (eds): Liver Pathology. Demos Medical, New York, NY, 2011.
22. Saber S, Goda R, El-Tanbouly GS and Ezzat D: Lisinopril inhibits nuclear transcription factor kappa B and augments sensitivity to silymarin in experimental liver fibrosis. Int Immunopharmacol 64: 340-349, 2018.

23. Domitrović $\mathrm{R}$ and Jakovac $\mathrm{H}$ : Effects of standardized bilberry fruit extract (Mirtoselect ${ }^{\circledR}$ ) on resolution of $\mathrm{CCl} 4$-induced liver fibrosis in mice. Food Chem Toxicol 49: 848-854, 2011.

24. Tsai JH, Liu JY, Wu TT, Ho PC, Huang CY, Shyu JC, Hsieh YS, Tsai CC and Liu YC: Effects of silymarin on the resolution of liver fibrosis induced by carbon tetrachloride in rats. J Viral Hepat 15: 508-514, 2008.

25. Hernandez-Gea V and Friedman SL: Pathogenesis of liver fibrosis. Annu Rev Pathol 6: 425-456, 2011.

26. Zhan L, Huang C, Meng XM, Song Y, Wu XQ, Yang Y and Li J: Hypoxia-inducible factor-1alpha in hepatic fibrosis: A promising therapeutic target. Biochimie 108: 1-7, 2015.

27. Li J, Li X, Xu W, Wang S, Hu Z, Zhang Q, Deng X, Wang J, Zhang J and Guo C: Antifibrotic effects of luteolin on hepatic stellate cells and liver fibrosis by targeting AKT/mTOR/p70S6K and TGFb/Smad signalling pathways. Liver Int 35: 1222-1233, 2015.

28. Park JH, Yoon J, Lee KY and Park B: Effects of geniposide on hepatocytes undergoing epitheliale-mesenchymal transition in hepatic fibrosis by targeting $\mathrm{TGFb} / \mathrm{Smad}$ and ERK-MAPK signaling pathways. Biochimie 113: 26-34, 2015.

29. Yang JH, Kim SC, Kim KM, Jang CH, Cho SS, Kim SJ, Ku SK, Cho IJ and Ki SH: Isorhamnetin attenuates liver fibrosis by inhibiting TGF- $\beta /$ Smad signaling and relieving oxidative stress. Eur J Pharmacol 783: 92-102,2016.

30. Friedman SL: Molecular regulation of hepatic fibrosis, an integrated cellular response to tissue injury. J Biol Chem 275: 2247-2250, 2000 .

31. Borkham-Kamphorst E, Herrmann J, Stoll D, Treptau J, Gressner AM and Weiskirchen R: Dominant-negative soluble PDGF-beta receptor inhibits hepatic stellate cell activation and attenuates liver fibrosis. Lab Invest 84: 766-777, 2004

32. Tahashi Y, Matsuzaki K, Date M, Yoshida K, Furukawa F, Sugano Y, Matsushita M, Himeno Y, Inagaki Y and Inoue K: Differential regulation of TGF-beta signal in hepatic stellate cells between acute and chronic rat liver injury. Hepatology 35: 49-61, 2002.

33. Marra F, Galastri S, Aleffi S and Pinzani M: Stellate cells. In: Dufour JF, Clavien PA: (Eds.), Signaling pathways in liver diseases, Springer-Verlag, Berlin, Heidelberg, 2010, pp. 41-68.

34. Friedman SL: Mechanisms of disease: Mechanismsofhepatic fibrosis and therapeutic implications. Nat Clin Pract Gastroenterol Hepatol 1: 98-105, 2004.

35. Gauldie J, Bonniaud P, Sime P, Ask K and Kolb M: TGF-beta, Smad3 and the process of progressive fibrosis. Biochem Soc Trans 35: 661-664, 2007

36. Parsons CJ, Takashima M and Rippe RA: Molecular mechanisms of hepatic fibrogenesis. J Gastroenterol Hepatol 22 (Suppl 1): S79-S84, 2007.

37. Feng XH and Derynck R: Specificity and versatility in tgf-beta signaling through smads. Annu Rev Cell Dev Biol 21: 659-693, 2005.

38. Cho HJ, Baek KE, Saika S, Jeong MJ and Yoo J: Snail is required for transforming growth factor-beta-induced epithelial-mesenchymal transition by activating PI3 kinase/Akt signal pathway. Biochem Biophys Res Commun 353: 337-343, 2007.

39. Burch ML, Zheng W and Little PJ: Smad linker region phosphorylation in the regulation of extracellular matrix synthesis. Cell Mol Life Sci 68: 97-107, 2011

40. Matsuura I, Wang G, He D and Liu F: Identification and characterization of ERK MAP kinase phosphorylation sites in Smad3. Biochemistry 44: 12546-12553, 2005.

41. Kim YS, Kim J, Kim KM, Jung DH, Choi S, Kim CS and Kim JS: Myricetin inhibits advanced glycation end product (AGE)-induced migration of retinal pericytes through phosphorylation of ERK1/2, FAK-1, and paxillin in vitro and in vivo. Biochem Pharmacol 93: 496-505, 2015.

42. Lechuga CG, Hernández-Nazara ZH, Domínguez Rosales JA, Morris ER, Rincón AR, Rivas-Estilla AM, Esteban-Gamboa A and Rojkind M: TGF-beta1 modulates matrix metalloproteinase-13 expression in hepatic stellate cells by complex mechanisms involving p38MAPK, PI3-kinase, AKT, and p70S6k. Am J Physiol Gastrointest Liver Physiol 287: G974-G987, 2004.

This work is licensed under a Creative Commons Attribution-NonCommercial-NoDerivatives 4.0 International (CC BY-NC-ND 4.0) License. 